\title{
Plant Invasion by Chromolaena Odorata Alters Soil Microbiome and Provides Insight Into the Role of the Fcb Group of Bacteria.
}

Lini N ( $\square$ lininirmala@gmail.com )

Mar Ivanios College https://orcid.org/0000-0002-3086-0883

Rani Jalajakumari Reghu

Manoj Narayanan

Ramachandran Sarojini Santhosh

Shiburaj Sugathan

Abhirami Ajith Kumar Chithra

Abhijit Joy Kumar Sophy

\section{Research Article}

Keywords: Plant invasion, soil metagenomics, microbiome, Bacteroidetes

Posted Date: January 28th, 2022

DOI: https://doi.org/10.21203/rs.3.rs-1285524/v1

License: (a) (i) This work is licensed under a Creative Commons Attribution 4.0 International License. Read Full License 


\section{Abstract}

\section{Purpose}

Plant invasion provides an excellent working model for understanding the effects of soil microbial communities associated with natural plant populations. In this study, we investigated the diversity and abundance of soil microbial communities of Chromolaena odorata, one of the most invasive weeds, and Tridax procumbent, a native plant in the same family.

\section{Methods}

Elemental analysis of carbon, hydrogen and nitrogen in soil samples was performed using a $\mathrm{CHN}$ analyzer. Meta-genome analyses were performed using Oxford Nanopore sequencing. The data assembly, analysis, and classifications of all functional categories were performed using the SqueezeMeta v1.0.0 Pipeline.

\section{Results}

There is an increase in $\mathrm{pH}$ along with an increase in $\mathrm{C}, \mathrm{H}$, and $\mathrm{N}$ in the soil of the invasive plant $C$. odorata. Copiotrophic microbiome along with the gene families responsible for transport, metabolism of carbohydrates and cellular processes are more abundant in $C$. odorata soil. In contrast, oligotrophic microbial communities along with gene families involved in energy production and cellular maintenance are more abundant in the T. procumbens soil.

\section{Conclusion}

Our studies show the association of plant invasion with an increase in nutrient cycling and foster the development of copiotrophic organisms, including Bacteroides. This is associated with an increase in carbohydrate metabolism and substrate utilization, as well as activation of pathways for stress adaptation and resistance.

\section{Introduction}

Chromolaena odorata (L.) King and Robinson (C. odorata) are some of the most widely distributed tropical shrubs. As one of the most invasive weeds in the world, it is a serious problem in Central and West Africa, India, Australia, Pacific Islands, and Southeast Asia (McFadyen 2003). It copes with a broad range of climates and has spread to major continents like Asia. When established, it can create pure stands in disturbed areas, grasslands, fallows, and forest plantations (Kriticos et al. 2005). A few studies have been conducted on the invasion of $C$. odorata. Humid climates are preferable to the establishment and growth of $C$. odorata, and that tropical humid regions with latitudes between $9^{\circ} 30^{\prime}$ and $10^{\circ} \mathrm{N}$ are likely to be suitable habitats for $C$. odorata under future climate conditions (Fandohan et al. 2015). Invasion among natural communities by these introduced species poses a major threat to biodiversity (Panetta and Gooden 2017). Worldwide Diverse native plant communities are difficult to re-establish on soils that 
were once harboured by invasive plants (Van der Putten et al. 2013). In India, 1,599 alien plant species belong to 841 genera and 161 families, making alien flora $8.5 \%$ of India's vascular flora (Reshi and Khuroo 2012). There are several hypotheses to explain alien species invasions, the prominent of which focus on the role of habitat disturbance, competitors and consumers, soil nutrient cyclin (Chabrerie et al. 2019). Many studies have focused on identifying traits that promote plant invasiveness. The influence of soil microbial communities on invasion by these plants needs investigation. Identifying the potential mechanisms that allow invasive species to thrive is essential for controlling existing and future invasions. The soil microbiota changes over time and plays an important role in determining plant productivity and species diversity. In recent years, the interaction between soil microbes and invaded plants has gained increasing attention (Chernov and Zhelezova 2020). Species introduced into an ecosystem can alter edaphic factors, altering the structure and functioning of the microbial community (Bell et al. 2020). However, it remains unclear how microbes contribute to the invasive process.

With the rapid and continuous development of cultivation-independent metagenomic approaches, our knowledge of soil microbes has increased (Neelakanta and Sultana 2013). Microbial communities in native soils consist of a few dominant species and many other rare taxa (Jousset et al. 2017). Deep metagenomic sequencing enables understanding genomic insights into a low abundance population and may reveal a wide range of plant-microbe interactions in soil. By comparing the soil microbiome of invasive and native species, the difference between the microbial composition of these two communities may enable better explanation, which would contribute to understanding the role of microbial communities in the mechanisms underlying $C$. odorata invasion. The extensive spread of $C$. odorata species and its suitability for climate change requires immediate attention to elucidate the mechanism of invasion and the contributing factors. In this work, the soil properties of $C$. odorata and its microbiota were studied. Tridax procumbens, a native plant of the Asteraceae family, was used as a control.

\section{Materials And Methods}

\section{Sample collection and analysis}

We collected soil samples from $C$. odorata and T. procumbens plants growing in the local areas of Nalanchira (8.5392 N 76.94 E), Thiruvananthapuram Kerala, India. Soil samples beneath five plants at a depth of $0-10 \mathrm{~cm}$ and a radius of $20 \mathrm{~cm}$ were collected and immediately stored at $-80{ }^{\circ} \mathrm{C}$ until analysis. Elemental analysis of carbon, hydrogen, and nitrogen was performed using a $\mathrm{CHN}$ analyzer $(2400 \mathrm{CHN}$ elemental analyzer from Perkin Elmer) according to the manufacturer's instructions.

\section{DNA extraction}

We have performed the extraction of DNA from soil samples using a commercial kit (Purelink microbiome, Thermo Fischer) designed to extract DNA from soil samples. Following the manufacturer's instructions, DNA was extracted by bead beating and purified by column chromatography. The extracted DNA was quantified using the Qubit instrument (Thermo Fischer). After staining with Ethidium Bromide, 
resolution on a $0.8 \%$ agarose gel was used to analyze the quality of the extracted DNA. DNA was stored at $-20^{\circ} \mathrm{C}$ until further processing. To avoid contamination, all procedures were performed aseptically.

\section{DNA sequencing and bioinformatics analysis}

Raw sequence data generated from the nanopore platform were filtered and assessed quality with the nanoQC software used for nanopore sequence data quality checking. Soil metagenome analysis of $C$. odorata and T. procumbens was performed using SqueezeMeta v1.0.0 Pipeline (Tamames and PuenteSánchez 2019), which can be run in three different modes. The first mode is the sequential mode, where the sequenced metagenome data are treated individually and analyzed. The second mode is the coassembly mode, where the sequenced reads are pooled to perform a single assembly, and the third is the merged mode, where a single set of contigs is generated from the assembled data. The sequenced samples were assembled to maximize the robustness of contig construction using MEGAHIT assembler (Li et al. 2015)

with default parameters and the resulting contigs were merged into a single co-assembly. MEGAHIT assembler assembles the whole data with no pre-processing steps, like partitioning and normalization. It produces longer contig N50 and average contig length with large reads aligned to the assembly. Contig statistics were done using prinseq (Schmieder and Edwards 2011). Redundant contigs were removed using CD-HIT (Schmieder and Edwards 2011). Contigs were merged using Minimus2 (Treangen et al. 2011).

The contigs were annotated to identify the function and taxonomic affiliations. Here, contigs corresponding to the same organism are identified using different properties of their sequences, such as composition or abundance, and clustered into genome bins. Binning was done by the software MaxBin2 inside the SqueezeMeta Pipeline. After this step, samples were mapped against assembled contigs and were used to calculate taxonomic and functional statistics. Gene prediction from the contigs was performed with Prodigal (Hyatt et al. 2010) and redundant contigs were removed using CD-HIT (Schmieder and Edwards 2011). SqueezeMeta Pipeline makes use of bioinformatics software like Diamond for the comparison of the gene sequences against other taxonomical and functional databases (Buchfink et al. 2015). All the functional analyses were carried out using SqueezeMeta Pipeline using the NCBI NR database, KEGG, and COG database Top Phyla and Class based on the taxonomy were identified for the contigs of the co-assembled whole genome. Interactive taxonomy distribution charts representing the complete microbial diversity in $C$. odorata and $T$. procumbens samples were developed using Krona tools (Ondov et al. 2011). The predicted genes were classified by the blast search against the PFAM-HMMER3 database (Finn et al. 2014). Coverage and abundance estimation of the genes and contigs were performed with Bowtie2 (Langmead and Salzberg 2012).

\section{Results}

\section{Soil physicochemical properties}


The soil sample of $C$. odorata is more acidic than that of $T$. procumbens. $C$. odorata soil had a $\mathrm{pH}$ of 5.4 and T. procumbens soil had a $\mathrm{pH}$ of 6.8. CHN analyses were conducted to determine the composition of carbon $(\mathrm{C})$, hydrogen $(\mathrm{H})$, and nitrogen $(\mathrm{N})$. The results showed that $\mathrm{C}, \mathrm{H}$, and $\mathrm{N}$ were abundant in the soil samples of $C$. odorata compared to T. procumbens soil (Table 1). Compared to the T. procumbens $(0.90 \%)$, the soil of $C$. odorata contains the highest percentage of $\mathrm{C}(9.78 \%)$. The $\mathrm{H}$ content is $0.28 \%$ less than $T$. procumbens soil $(0.48 \%)$. The $\mathrm{N}$ content of the $C$. odorata soil is $0.74 \%$ and that of the $T$. procumbens soil is $0.18 \%$.

Table. 1 CHN elemental analysis of soil

\begin{tabular}{|cllll|}
\hline Run & Weight & Carbon & Hydrogen & Nitrogen \\
CM & 1.331 & $9.78 \%$ & $2.08 \%$ & $0.74 \%$ \\
TRX & 1.374 & $0.90 \%$ & $0.48 \%$ & $0.18 \%$ \\
\hline STD & 1.378 & $71.19 \%$ & $6.79 \%$ & $10.31 \%$ \\
\hline
\end{tabular}

\section{Bioinformatics analysis}

Based on microbial genome sequencing using nanopore sequencing, 2923 raw reads were generated for the $C$. odorata soil sample and 2350 raw reads for $T$. procumbens soil. The quality of the raw data was checked using the nanoQC software program for quality checking of nanopore sequencing data. In both cases, the quality of the raw data was satisfactory. Figures $1 \mathrm{a}$ and $1 \mathrm{~b}$ illustrate the quality plots based on the Phred scores. The average Phred score of the sequencing reads for the samples was calculated as $\geq 20$. Megahit was used to assemble samples individually, followed by the merging of the resulting contigs into a single co-assembly. The total assembly statistics for the co-assembled genome comprise 684256 bases and 312 reads shown in supplementary Table 1.

\section{Taxonomy and distribution}

Taxonomic analysis of the $C$. odorata soil revealed that 99 reads mapped to bacteria, 7 reads mapped to Eukaryota, and 65 reads mapped to an unknown taxon. For the T. procumbens soil sample, 78 reads mapped to bacteria, 24 reads mapped to Eukaryota, and 40 reads mapped to unknown taxa. In the interactive taxonomic distribution of total microbial diversity based on contig taxonomy, the Krona data shows that the $C$. odorata soil contains $58 \%$ bacteria, $4 \%$ eukaryotes, and $38 \%$ unknown species. Among the bacterial species, $28 \%$ are Terrabacteria, $26 \%$ are Proteobacteria, $14 \%$ are FCB bacteria and $30 \%$ are other bacteria. Actinobacteria make up $71 \%$ of the Terra bacteria. Bacteroidetes make up $79 \%$ of the FCB group bacteria. In the T. procumbens soil samples, Proteobacteria accounted for $24 \%$ of the total bacterial population, $38 \%$ of Terrabacteria and $4 \%$ of Acidobacteria. Among the Proteobacteria, $32 \%$ were Beta Proteobacteria, $16 \%$ were Alpha Proteobacteria, and $11 \%$ were Delta Proteobacteria. The Terrabacteria comprised 53\% Actinobacteria, 13\% Chloroflexia and 10\% Tenericutes (Figure. 2a and 2b). 
From the taxonomy assigned to the contigs of the co-assembled whole-genome metagenome, the distribution of the top Phyla and Class are shown in Figure 3 and Figure 4. The results show that Proteobacteria and Actinobacteria are more abundant in C. odorata soils compared to T. procumbens soils. In addition, the Bacteroidetes were found in large numbers in $C$. odorata soil. The phylum Chlorofelxi, Planctomycetes, Acidobacteria, Arthropoda, and Tenericutes, and Tenericutes are abundant in T. procumbens soils. Among Proteobacteria, Alpha Proteobacteria, and Gamma Proteobacteria classes were more abundant in $C$. odorata soils, while Beta Proteobacteria and Delta Proteobacteria were more abundant in T. procumbens soils. Species of Ignavibacteria were common within the class of Saprospiria. Chordates were observed among eukaryotes in $C$. odorata soils. However, Cloroflexi and insects were abundant in the T. procumbens soil.

\section{Functional distribution}

A full functional annotation was performed using the NCBI NR database, the KEGG database, and the COG database. The overall statistics of the ORFs detected and their functional distribution are presented in supplementary Table 2. The statistics for ORFs identified in the assembled contigs showed that 1019 ORFs were mapped, 328 ORFs in C. odorata soil samples and 278 ORFs in T. procumbens soil samples. Aragorn predicted three ORFs (Assembly-7, Chrombleana-11, and Tridax-1). The mapping of metagenomic ORFs to the databases of orthologous gene groups (COG and $\mathrm{KO}$ ) revealed many enriched functional features. From the KEGG annotation, 220 ORFs were mapped into the assembly, 71 ORFs in $C$. odorata soil and 57 ORFs in T. procumbens soil. From COG -annotation, assembly 335, the C. odorata 112 ORFs and the T. procumbens 57 ORFs. Based on PFAM annotation, 103 ORFs have been assigned to assembly, 34 ORFs in C. odorata soil and 30 ORFs in T. procumbens soil (Figure.6).

According to KEGG annotation, metagenomic ORFs were aligned with the genes associated with 145 metabolic pathways. Among the functional categories in KEGG, genes involved in metabolism are the most abundant in $C$. odorata soil and T. procumbens soil. Interestingly, carbohydrate metabolism was the most abundant metabolic pathway (31\%) in $C$. odorata soils. With $C$. odorata plants, the second abundant category was involved in cellular processes. It is noteworthy that in the cellular process; the genes associated with amino acid transporters were abundant in $C$. odorata soils. There was an increase in pathways associated with genetic information and processing in Chromolaena species (Fig.5a)

Besides this, the most abundant functional categories of the gene were Branched-chain amino acids, $A B C$ transporter-periplasmic leucine binding subunit livK (K0199) and its orthologues genes in C. odorata soil. Glutathione S transferase (K00799), Fe-S assembly proteins (K09014) were also abundant in the $C$.

odorata soil sample (Fig.5b). In T. procumbens sample Elongation factor G (K02355), the large subunit of ribosomal protein L13 (K02871), Monovalent cation H+ antiporter (K03316) was the prominent category. In COG category glycosyl transferase (COG 0435), ABC-Type transporter system involved in Fe-S cluster assembly permease component (COG 0719) ABC transporter substrate-binding protein (ENOG410XNWR), Glusamylase and related glycosyl hydrolases (COG3387) were the abundant categories. With $T$. 
procumbens plants signal transduction histidine kinase (COG0642) FOG: CheY-like receiver (COG0784) Ribosomal protein L13 (COG0102) Translation Elongation factor (COG0480) was more abundant.

\section{Discussion}

Biological invasions have been identified as a growing threat to global sustainability, and their impact on soil microbial communities has attracted much attention in recent years. $C$. odorata has a worldwide distribution, in neotropical areas affected by human activities. Once introduced, $C$. odorata can become dominant within a short period (McFadyen and Skarratt 1996). C. odorata can increase soil nutrient levels (Wei et al. 2017) and inhibit native plants (Mangla et al. 2008). Given the wide distribution of this $C$. odorata species and its ability to adapt to climate change, it is imperative to understand the factors that influence its invasion mechanisms.

Plant growth is promoted by the interplay of soil microbes and the physiological environment. In our study, we analyzed soil physicochemical factors and microbial diversity in soil samples of $C$. odorata and compared them with the soil of the native plant T. procumbens. We collected both soil samples from the same location. Soil pH can be considered a key variable due to its influence on microbial activity, nutrient availability, and plant growth. Our results show that soil samples of $C$. odorata are more acidic than those of $T$. procumbens, which may be due to the acidic extrudates generated by the invasive plants (Ikhajiagbe 2016). There is growing evidence that soil nutrient availability is a major abiotic factor that can influence the success of alien invasive plants. An alien invasive plant usually shows higher efficiency in nutrient utilization and greater flexibility in nutrient management compared to its native counterpart (Osborne and Gioria 2018). Analysis of $\mathrm{CHN}$ shows that the soil of $C$. odorata contains a higher concentration of $\mathrm{C}, \mathrm{H}$ and $\mathrm{N}$ than that of $T$. procumbens. This may lead to nutrient-rich conditions in $C$. odorata soils, which promote nutrient cycling and accelerate the growth of invasive plants. This is consistent with previous reports showing that the amount of $\mathrm{C}$ and $\mathrm{N}$ in soil appears to increase with the severity of $C$. odorata invasion (Wei et al. 2017). Invasive plants effectively participate to accelerate soil carbon and nitrogen cycling (Jo et al. 2017). For example, Bromus tectorum invasion increases microbial activity in the arid grasslands in the southeastern United States and stimulates microbial nitrogen cycling (Schaeffer et al. 2012). Organic carbon is a measurable attribute of soil organic matter. Organic matter is important for nutrient storage and turnover, soil structure, moisture retention and availability, pollutant degradation, and carbon sequestration. Sites with greater $\mathrm{C}$ and $\mathrm{N}$ storage are associated with high bacterial concentrations and high cation exchange capacities (Caspi et al. 2019). Thus, these results suggest that acidic $\mathrm{pH}$, along with nutrient cycling, plays an important role in controlling $C$. odorata invasion.

\section{Comparison of the microbiome and functional analysis of soil microbial communities}

Actinobacteria and Proteobacteria, the copiotrophic bacterial groups, were the most abundant microbial groups identified in C. odorata soil samples. Acidobacteria and Chloroflexi were more abundant in the soil of T. procumbens. Proteobacteria are a phylum of Gram-negative bacteria that live in soil and can perform functions related to carbon, nitrogen, and sulfur cycling (Zhou et al. 2020). Acidobacteria 
belongs to the oligotrophic group of bacteria that live in nutrient-poor and acidic environments. Previous reports on the analysis of the soil microbiome of invasive plants also indicated Proteobacteria, Actinobacteria, and Ascomycota (Rodríguez-Caballero et al. 2020).

An analysis of the rhizospheric microbiome in Thailand shows that these bacteria are also abundant in $C$. odorata soils (Ruangdech et al. 2017). Actinobacteria, Alpha Proteobacteria, and Gamma Proteobacteria may be significantly enriched in soils with N and NP (Shivlata and Tulasi 2015; Jung et al. 2016; Rodríguez-Caballero et al. 2020). Our results support the hypothesis that copiotrophic groups (e.g., Proteobacteria and Actinobacteria) with fast growth rates increase under nutrient-rich conditions, whereas oligotrophic groups (e.g., Acidobacteria and Chloroflexi), which have slower growth rates, decrease (Fierer et al. 2005). This shift in microbial community structure associated with a nutrient-rich environment may play an important role in plant invasion. In soils treated with artificial water rich in organic and mineral loads, the abundance of Gamma Proteobacteria, Cytophagia, Saprospiria and Sphingobacteria increased. Similarly, we have observed the abundance of these bacterial genera in soil samples with invasive plant $C$. odorata.

We also observed a significant decrease in arthropod communities in $C$. odorata soils. This correlates with earlier reports that show a reduction in the arthropod community during invasion (van Hengstum et al. 2014). In many ecosystems, arthropods are the most important primary consumers and necessary for the pollination and dispersal of a variety of plant species (Chapman and Chapman 1998).

Evidence suggests that invaders negatively affect the visitation and reproductive success of native cooccurring plants (Morales and Traveset 2009), and invaders may even disrupt the mutualistic relationships between native species and insects (Traveset et al. 2013). The response of arthropods to plant invasions should be of particular concern given their abundance, diversity and indispensable roles as herbivores, pollinators, predators and prey.

\section{Importance of FCB group of bacteria in plant invasion}

We detected an abundance of bacteria belonging to the FCB group in the soils of $C$. odorata. The FCB group comprises Fibrobacteres, Chlorobi and Bacteroidetes. Bacteroidetes occur in many ecosystems and play an important role in the degradation of polymeric organic matter (Zheng et al. 2021). Because of their high abundance, their ability to degrade polysaccharides, and their diverse enzyme systems, Bacteriodates play an important role in the carbon and nutrient cycles (Gupta 2004). They have an effective genomic organization in which most of their CAZymes are localized in the polysaccharide utilization loci. Carbohydrate active enzymes (CAZymes) are responsible for the synthesis, modification, and degradation of carbohydrate biopolymers in plants. According to our results, the genes involved in carbohydrate metabolism were abundant in the soil of $C$. odorata. This indicates that copiotroph organism like Bacteroidetes which produce a variety of carbohydrate-active enzymes (Larsbrink and McKee 2020) plays a major role utilization of plant-derived polysaccharides and thereby accelerating the growth of invasive plants. Ignavibacteria, a member of the Bacteroidetes, have been isolated from hot water sediments from Yellowstone National Park and have been reported to metabolize nitrogen and 
aromatic compounds (Tian et al. 2015). Cytophaga Flavobacterium (CF), are bacteria that may cause soil degradation. Several of these organisms exist all over the world and are known for their role in degrading organic compounds. Studies have shown that they are present in the soil, especially in the root zone. In addition, these bacteria produce a wide range of hydrolytic enzymes that can degrade complex carbohydrates. This suggests that the FCB group play a crucial role in plant invasion. Because of their adaptability to changing conditions and substrate utilization, they threaten the ecological balance in changing climatic conditions that favour plant invasions. Ether-linked lipids in their membrane can help Bacteroidetes adapt to the different stress conditions they are exposed to during their development (Siliakus et al. 2017; Villanueva et al. 2021).

\section{Functional category}

Functional annotation shows that one of the most abundant genes expressed in the soil microbiome of C. odorata was the Branched-chain amino acids $A B C$ transporter- periplasmic leucine binding subunit livK and its orthologous genes. Studies suggest that catabolism of Branched-chain amino acids may contribute to plant resistance to pathogens by modulating the defence pathways (Zeier 2013). The second most abundant category was the Fe-S cluster assembly protein SufB FE -S. It is necessary for the proper functioning of several metabolic pathways and resistance to genotoxic and abiotic stresses. In addition, they are involved in resistance to non-host plants and plant immunity (Fonseca et al. 2020). Iron and sulfur (Fe-S) clusters are essential for several biochemical processes, including respiration, photosynthesis and nitrogen fixation (Gao 2020)

Bacterial glutathione transferase (GST) is another functional category that is over-expressed. Bacterial GSTs mediate several processes, including degradation of xenobiotics, defence against chemical and oxidative stress, and resistance to antimicrobial agents. In addition, GSTs play a role in many metabolic processes including, the degradation of lignin, the biotransformation of dichloromethane, and the reductive dechlorination of pentachlorophenol (Allocati et al. 2009). Glutathione transferases (GSTs) are ancient, ubiquitous, multifunctional antioxidant enzymes that play important roles in plant growth and development. Recent studies suggest the involvement of GSTs in cell signalling kinases, ion channel formation and modulation, oxidation-reduction reactions, etc. GSTs can also act as ligands and are involved in the transport of auxins within cells (Kumar and Trivedi 2018). This indicates the involvement of genes in pathogen resistance and stress tolerance are involved more in invasive soil. Several oligopeptide importers, 3-isopropyl malate dehydrogenase, enzymes of amino acid biosynthesis and Elongation factor $\mathrm{G}$ are expressed in the soil of $T$. procumbens. These genes are involved in the transport of nutrients, cell signalling, amino acid biosynthesis and protein synthesis. A recent study using genome analyses also shows that gene families involved in the transport and metabolism of carbohydrates and amino acids often predominate in copiotrophic microorganisms. Oligotrophs exhibited a higher prevalence of gene families involved in energy production and conversion. Translation efficiency through codon optimization was an important factor in controlling the growth pattern of oligotrophs and copiotrophs. This is correlating with our results which show that the gene family responsible for transport, metabolism of carbohydrates and cellular processes are more prevalent in invasive soil 
microbial communities. However, gene families involved in energy production and environmental information and processing are more prevalent in the microbial community of $T$. procumbens.

These results and our findings suggest that copiotrophic organisms such as Bacteroides play an important role in plant cell invasion. Our studies show the association of plant invasion with an increase in nutrient cycling fosters the development of copiotrophic organisms. This is also associated with an increase in carbohydrate metabolism and substrate utilization, as well as activation of pathways for stress adaptation and resistance.

\section{Conclusion}

We found an increase in $\mathrm{pH}$ along with an increase in the availability of $\mathrm{C}, \mathrm{H}$, and $\mathrm{N}$ in the soil of $\mathrm{C}$. odorata, which could enhance a shift from a copiotrophic to an oligotrophic microbial community in the microbiome. The invasive plants might be able to alter soil conditions and re-establish soil microbial communities once they have invaded, thereby gaining access to soil nutrients more readily than their associated competitors. This could be accompanied by an increase in gene families involved in substrate utilization, adaptation to stress, and resistance to pathogens. Plant invasion can alter soil biochemical properties, nutrient cycling, and microbial diversity, creating an ecological imbalance. The development and application of a microbial consortium in conjunction with altering the nutrient-rich environment is an alternative strategy to promote the growth of native plant species. We present here a preliminary analysis of the meta-genome as a basis for further study. Further studies are needed to fully understand the mechanisms of invasive plants and their impact on environmental restoration.

\section{Declarations}

\section{Data Availability Statement}

The datasets presented in this study can be found in the online repository, https://www.ncbi.nlm.nih.gov/_with an accession number PRJNA746476

\section{Acknowledgement}

We would like to acknowledge Dr Zyju D P for reviewing and editing the manuscript.

\section{Author contribution}

All authors contributed to the intellectual input and supported this study and the preparation of the manuscript. the research and conducted the experiments. RJR and MN analyzed the data and RSS and SS performed the nanopore sequencing. AS and AJ helped with soil sample collection and $\mathrm{CHN}$ analysis. LN designed and supervised the work and approved the manuscript for publication. All authors read and approved the final manuscript.

\section{Funding}


This research received no specific grant from any funding agency in the public, commercial, or not-forprofit sectors

\section{Competing Interests}

The authors have no relevant financial or non-financial interests to disclose.

\section{References}

1. Allocati N, Federici L, Masulli M, Di llio C (2009) Glutathione transferases in bacteria. FEBS J 276:58-75

2. Bell JK, Siciliano SD, Lamb EG (2020) A survey of invasive plants on grassland soil microbial communities and ecosystem services. Sci Data 7:1-8

3. Buchfink B, Xie C, Huson DH (2015) Fast and sensitive protein alignment using DIAMOND. Nat Methods 12:59-60

4. Caspi T, Hartz LA, Soto Villa AE, et al (2019) Impacts of invasive annuals on soil carbon and nitrogen storage in southern California depend on the identity of the invader. Ecol Evol 9:4980-4993

5. Chabrerie O, Massol F, Facon B, et al (2019) Biological invasion theories: Merging perspectives from population, community and ecosystem scales

6. Chapman RF, Chapman RF (1998) The insects: structure and function. Cambridge university press

7. Chernov TI, Zhelezova AD (2020) The dynamics of soil microbial communities on different timescales: a review. Eurasian Soil Sci 53:643-652

8. Fandohan AB, Oduor AM, Sodé Al, et al (2015) Modeling vulnerability of protected areas to invasion by Chromolaena odorata under current and future climates. Ecosyst Health Sustain 1:1-12

9. Fierer N, Jackson JA, Vilgalys R, Jackson RB (2005) Assessment of soil microbial community structure by use of taxon-specific quantitative PCR assays. Appl Environ Microbiol 71:4117-4120

10. Finn RD, Bateman A, Clements J, et al (2014) Pfam: the protein families database. Nucleic Acids Res 42:D222-D230

11. Fonseca JP, Lee H-K, Boschiero C, et al (2020) Iron-Sulfur Cluster Protein NITROGEN FIXATION SLIKE1 and Its Interactor FRATAXIN Function in Plant Immunity. Plant Physiol 184:1532-1548

12. Gao F (2020) Iron-sulfur cluster biogenesis and iron homeostasis in cyanobacteria. Front Microbiol $11: 165$

13. Gupta RS (2004) The phylogeny and signature sequences characteristics of Fibrobacteres, Chlorobi, and Bacteroidetes. Crit Rev Microbiol 30:123-143

14. Hyatt D, Chen G-L, LoCascio PF, et al (2010) Prodigal: prokaryotic gene recognition and translation initiation site identification. BMC Bioinformatics 11:1-11

15. Ikhajiagbe $B$ (2016) Possible adaptive growth responses of Chromolaena odorata during heavy metal remediation. Ife J Sci 18:403-411 
16. Jo I, Fridley JD, Frank DA (2017) Invasive plants accelerate nitrogen cycling: evidence from experimental woody monocultures. J Ecol 105:1105-1110

17. Jousset A, Bienhold $C$, Chatzinotas $A$, et al (2017) Where less may be more: how the rare biosphere pulls ecosystems strings. ISME J 11:853-862

18. Jung J, Philippot L, Park W (2016) Metagenomic and functional analyses of the consequences of reduction of bacterial diversity on soil functions and bioremediation in diesel-contaminated microcosms. Sci Rep 6:1-10

19. Kriticos DJ, Yonow T, McFadyen RE (2005) The potential distribution of Chromolaena odorata (Siam weed) in relation to climate. Weed Res 45:246-254

20. Kumar S, Trivedi PK (2018) Glutathione S-transferases: role in combating abiotic stresses including arsenic detoxification in plants. Front Plant Sci 9:751

21. Langmead B, Salzberg SL (2012) Fast gapped-read alignment with Bowtie 2. Nat Methods 9:357359

22. Larsbrink J, McKee LS (2020) Bacteroidetes bacteria in the soil: Glycan acquisition, enzyme secretion, and gliding motility. Adv Appl Microbiol 110:63-98

23. Li D, Liu C-M, Luo R, et al (2015) MEGAHIT: an ultra-fast single-node solution for large and complex metagenomics assembly via succinct de Bruijn graph. Bioinformatics 31:1674-1676

24. Mangla S, Inderjit, Callaway RM (2008) Exotic invasive plant accumulates native soil pathogens which inhibit native plants. J Ecol 96:58-67

25. McFadyen RC (2003) Chromolaena in East Timor: history, extent and control. Chromolaena in the Asia-Pacific region. In: Proc. of the 6th International Workshop on Biological Control and Management of Chromolaena, Cairns, Australia. pp 8-10

26. McFadyen RC, Skarratt B (1996) Potential distribution of Chromolaena odorata (siam weed) in Australia, Africa and Oceania. Agric Ecosyst Environ 59:89-96

27. Morales CL, Traveset A (2009) A meta-analysis of impacts of alien vs. native plants on pollinator visitation and reproductive success of co-flowering native plants. Ecol Lett 12:716-728

28. Neelakanta G, Sultana H (2013) The use of metagenomic approaches to analyze changes in microbial communities. Microbiol Insights 6:MBI-S10819

29. Ondov BD, Bergman NH, Phillippy AM (2011) Interactive metagenomic visualization in a Web browser. BMC Bioinformatics 12:1-10

30. Osborne B, Gioria M (2018) Plant invasions

31. Panetta FD, Gooden B (2017) Managing for biodiversity: impact and action thresholds for invasive plants in natural ecosystems

32. Reshi ZA, Khuroo AA (2012) Alien plant invasions in India: Current status and management challenges. Proc Natl Acad Sci India Sect B Biol Sci 82:305-312

33. Rodríguez-Caballero G, Roldán A, Caravaca F (2020) Invasive Nicotiana glauca shifts the soil microbial community composition and functioning of harsh and disturbed semiarid Mediterranean 
environments. Biol Invasions 22:2923-2940

34. Ruangdech T, Wongphatcharachai M, Staley C, et al (2017) Influence of heavy metals on rhizosphere microbial communities of Siam weed (Chromolaena odorata (L.)) using a 16S rRNA gene amplicon sequencing approach. Agric Nat Resour 51:137-141

35. Schaeffer SM, Ziegler SE, Belnap J, Evans RD (2012) Effects of Bromus tectorum invasion on microbial carbon and nitrogen cycling in two adjacent undisturbed arid grassland communities. Biogeochemistry 111:427-441

36. Schmieder R, Edwards R (2011) Quality control and preprocessing of metagenomic datasets. Bioinformatics 27:863-864

37. Shivlata L, Tulasi S (2015) Thermophilic and alkaliphilic Actinobacteria: biology and potential applications. Front Microbiol 6:1014

38. Siliakus MF, van der Oost J, Kengen SW (2017) Adaptations of archaeal and bacterial membranes to variations in temperature, $\mathrm{pH}$ and pressure. Extremophiles 21:651-670

39. Tamames J, Puente-Sánchez F (2019) SqueezeMeta, a highly portable, fully automatic metagenomic analysis pipeline. Front Microbiol 9:3349

40. Tian M, Zhao F, Shen X, et al (2015) The first metagenome of activated sludge from full-scale anaerobic/anoxic/oxic (A20) nitrogen and phosphorus removal reactor using Illumina sequencing. J Environ Sci 35:181-190

41. Traveset A, Heleno R, Chamorro S, et al (2013) Invaders of pollination networks in the Galápagos Islands: emergence of novel communities. Proc R Soc B Biol Sci 280:20123040

42. Treangen TJ, Sommer DD, Angly FE, et al (2011) Next generation sequence assembly with AMOS. Curr Protoc Bioinforma 33:11-8

43. Van der Putten WH, Bardgett RD, Bever JD, et al (2013) Plant-soil feedbacks: the past, the present and future challenges. J Ecol 101:265-276

44. van Hengstum T, Hooftman DA, Oostermeijer JGB, van Tienderen PH (2014) Impact of plant invasions on local arthropod communities: a meta-analysis. J Ecol 102:4-11

45. Villanueva $L$, von Meijenfeldt FB, Westbye $A B$, et al (2021) Bridging the membrane lipid divide: bacteria of the FCB group superphylum have the potential to synthesize archaeal ether lipids. ISME J 15:168-182

46. Wei H, Xu J, Quan G, et al (2017) Invasion effects of Chromolaena odorata on soil carbon and nitrogen fractions in a tropical savanna. Ecosphere 8:e01831

47. Zeier J (2013) New insights into the regulation of plant immunity by amino acid metabolic pathways. Plant Cell Environ 36:2085-2103

48. Zheng R, Cai R, Liu R, et al (2021) Maribellus comscasis sp. nov., a novel deep-sea Bacteroidetes bacterium, possessing a prominent capability of degrading cellulose. Environ Microbiol 23:45614575 
49. Zhou Z, Tran PQ, Kieft K, Anantharaman K (2020) Genome diversification in globally distributed novel marine Proteobacteria is linked to environmental adaptation. ISME J 14:2060-2077

\section{Figures}

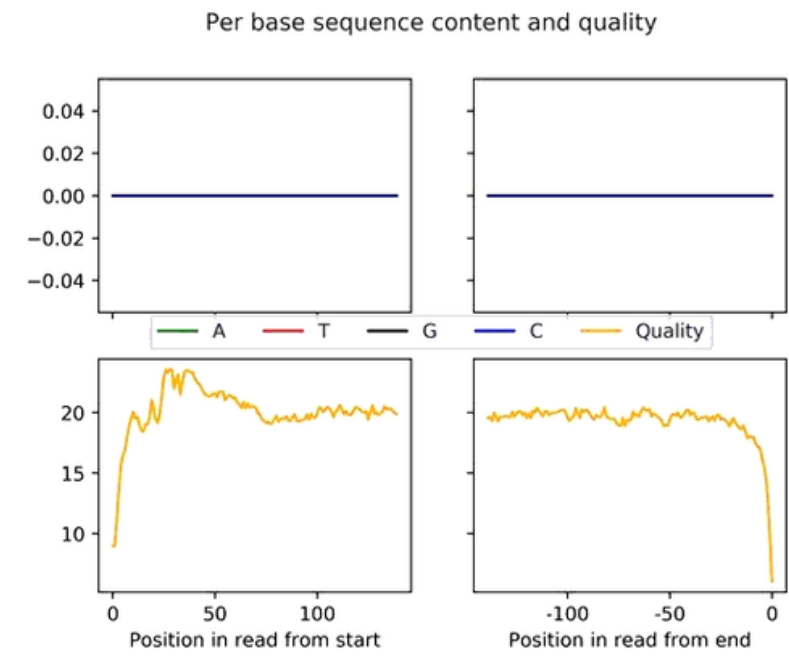

Figure 1(a) C. odorata

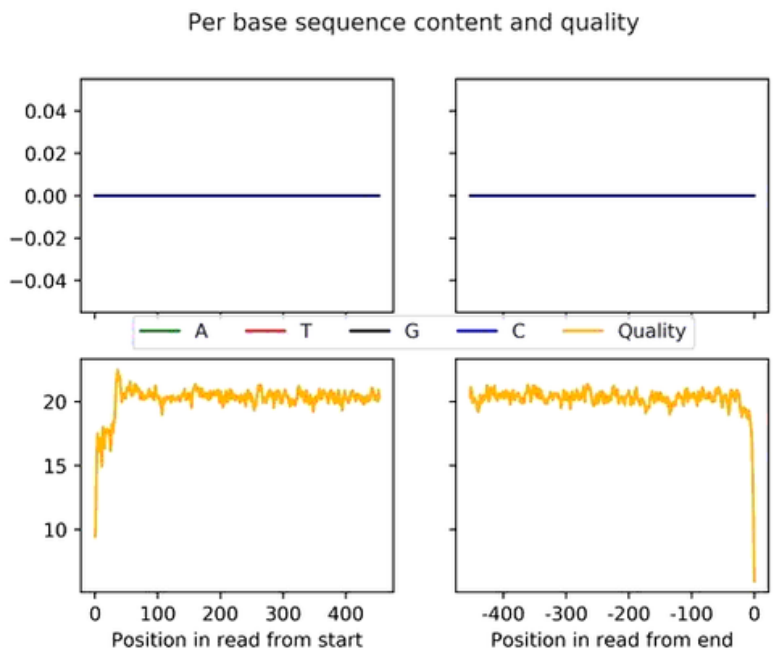

Figure 1(b) T. procumbens

\section{Figure 1}

Phred quality score values for (a) C. odorata soils sample and (b) T. procumbens soil sample. The raw data quality was checked with the nanoQC software used for nano sequence data quality checking. The $x$-axis displays the base position in the read, and the $y$-axis shows quality scores. 


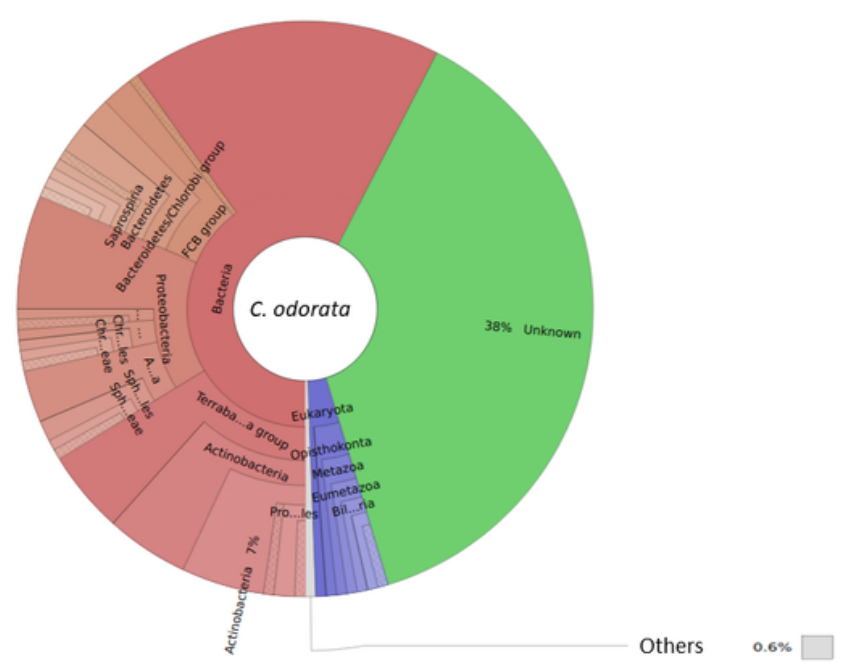

Figure 2(a)

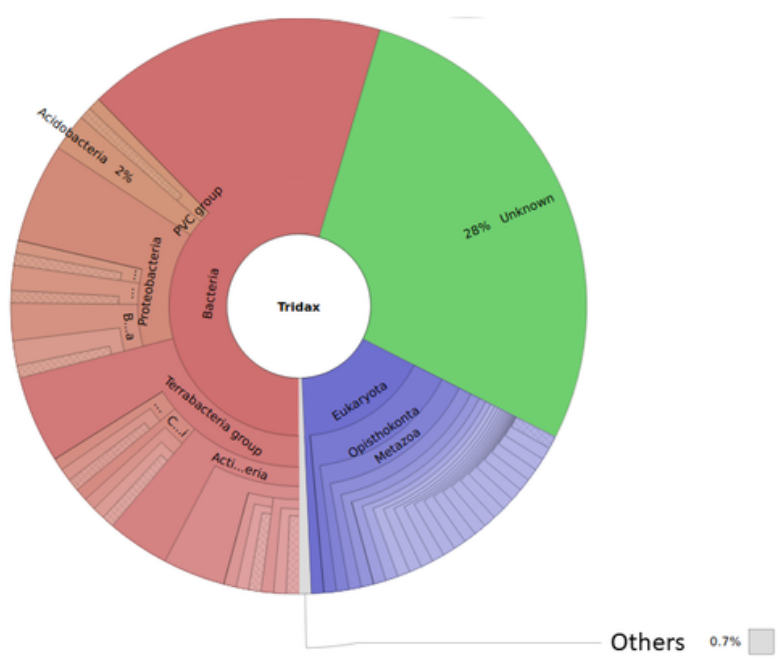

Figure 2(b)

\section{Figure 2}

a: Krona chart representing the taxonomic composition of the microbial community associated with $C$. odorata soil sample based on contig taxonomy.

b: Krona chart representing the taxonomic composition of the microbial community associated with the T. procumbens soil sample based on contig taxonomy.

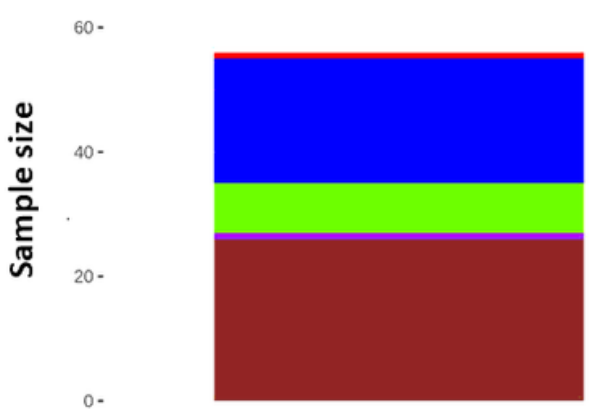

C. odorata

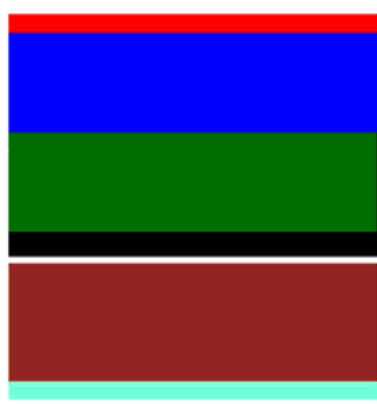

T. procumbens

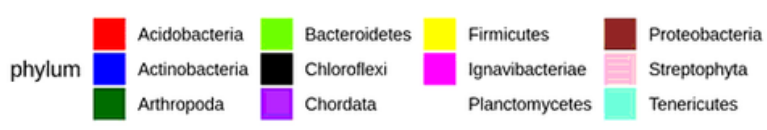

Figure 3

Figure 3 
Represents the distribution of the top Phyla in Chromblaena and Tridax soil samples. Proteobacteria (brown), Actinobacteria (blue) and Bacteroidetes (light green) are predominant in Chromblaena compared to Tridax. The phylum Chlorofelxi (black), Planctomycetes (white), Acidobacteria (red), Arthropoda (green), and Tenericutes (cyan) are abundant in T. procumbens soils.

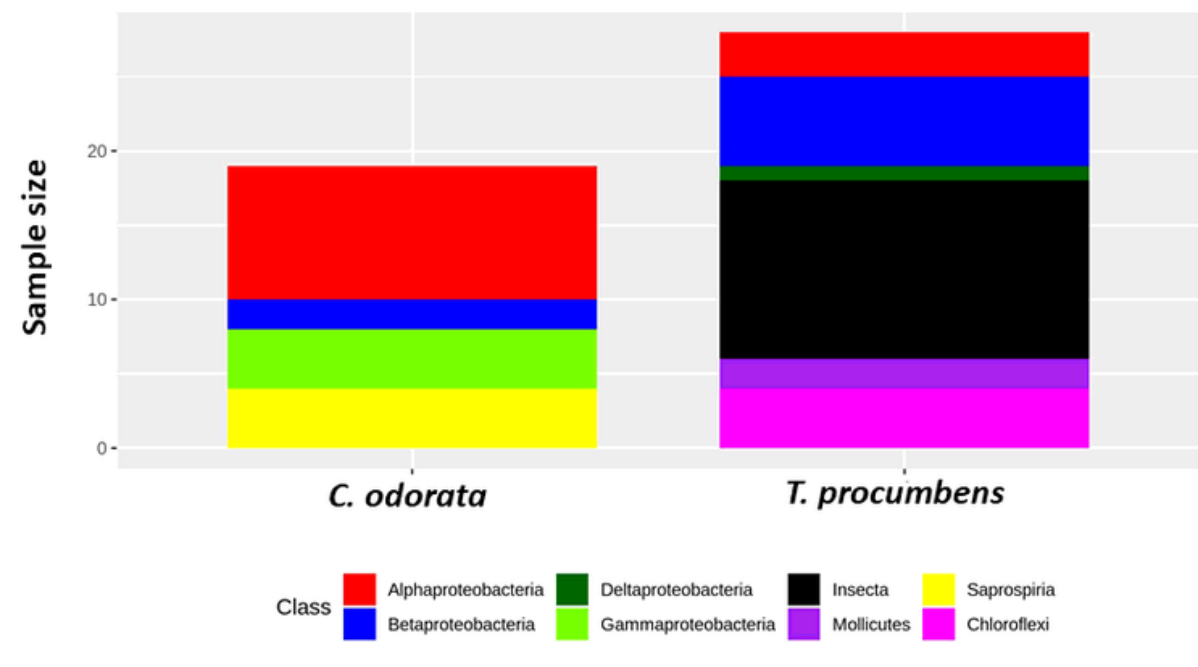

Figure 4

\section{Figure 4}

Represents the distribution of the top Class in Chromblaena and Tridax soil samples. Alpha Proteobacteria (red), and Gamma Proteobacteria (light green), Saprospiria (yellow) classes were more abundant in C. odorata soils, while Beta Proteobacteria (blue) and Delta Proteobacteria (green), Chloroflexi (Pink) are abundant in T. procumbens soils. 


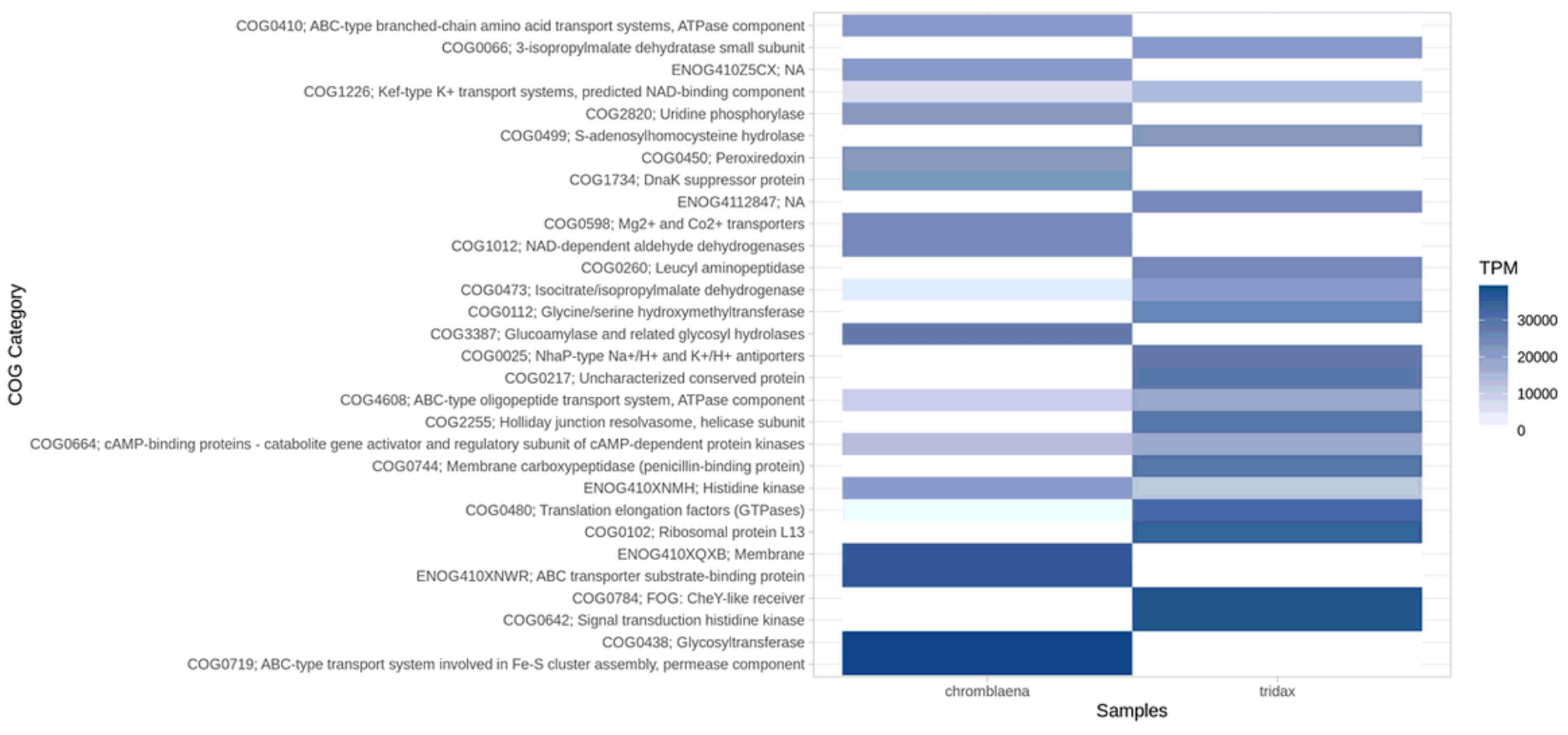

Figure 5

\section{Figure 5}

Top 30 enriched COG categories functional categories are represented in the form of the heat map. The colour key codes for abundance were observed based on TPM-Transcripts per million Values. 


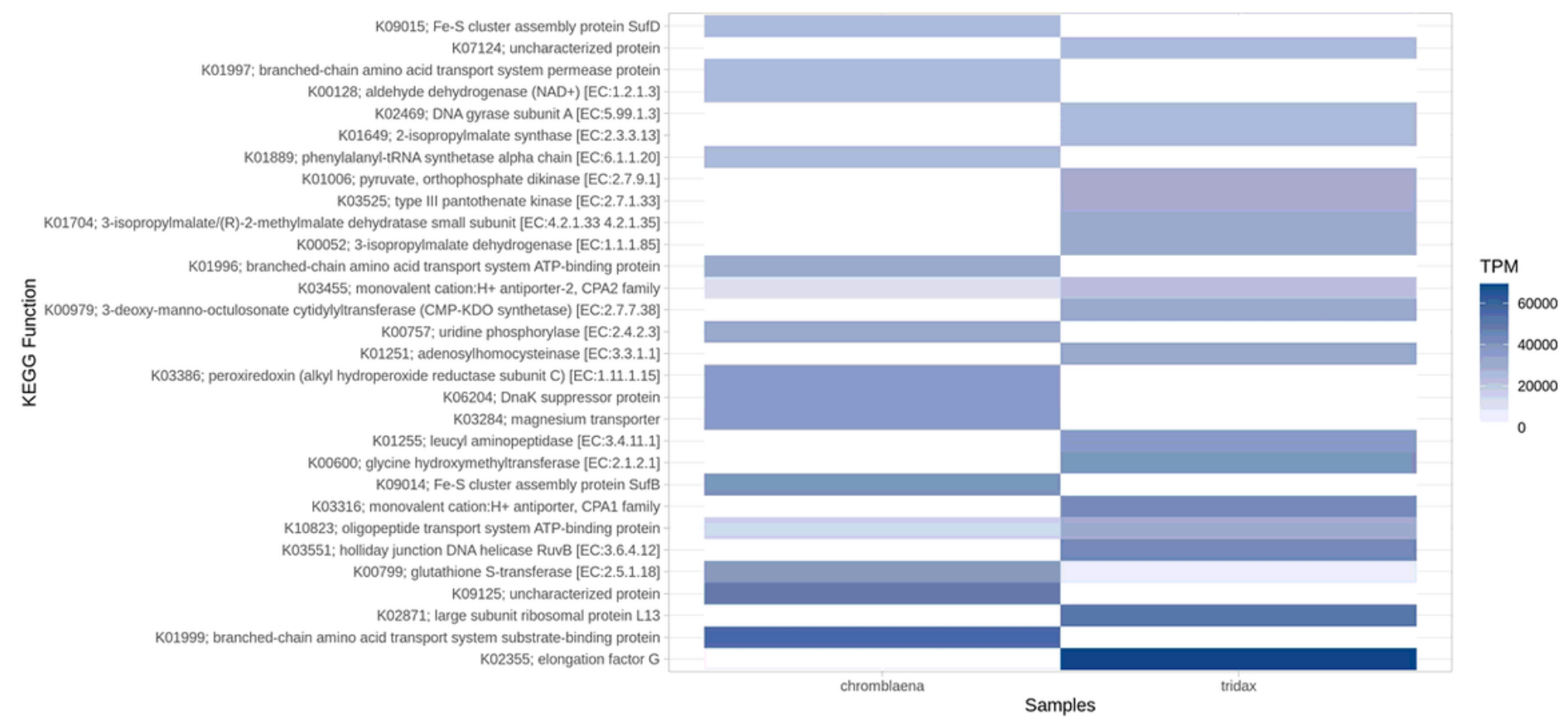

Figure 6

\section{Figure 6}

Top 30 enriched KEGG categories are represented in the form of the heat map. The colour key codes for abundance were observed based on TPM-Transcripts per million Values. 


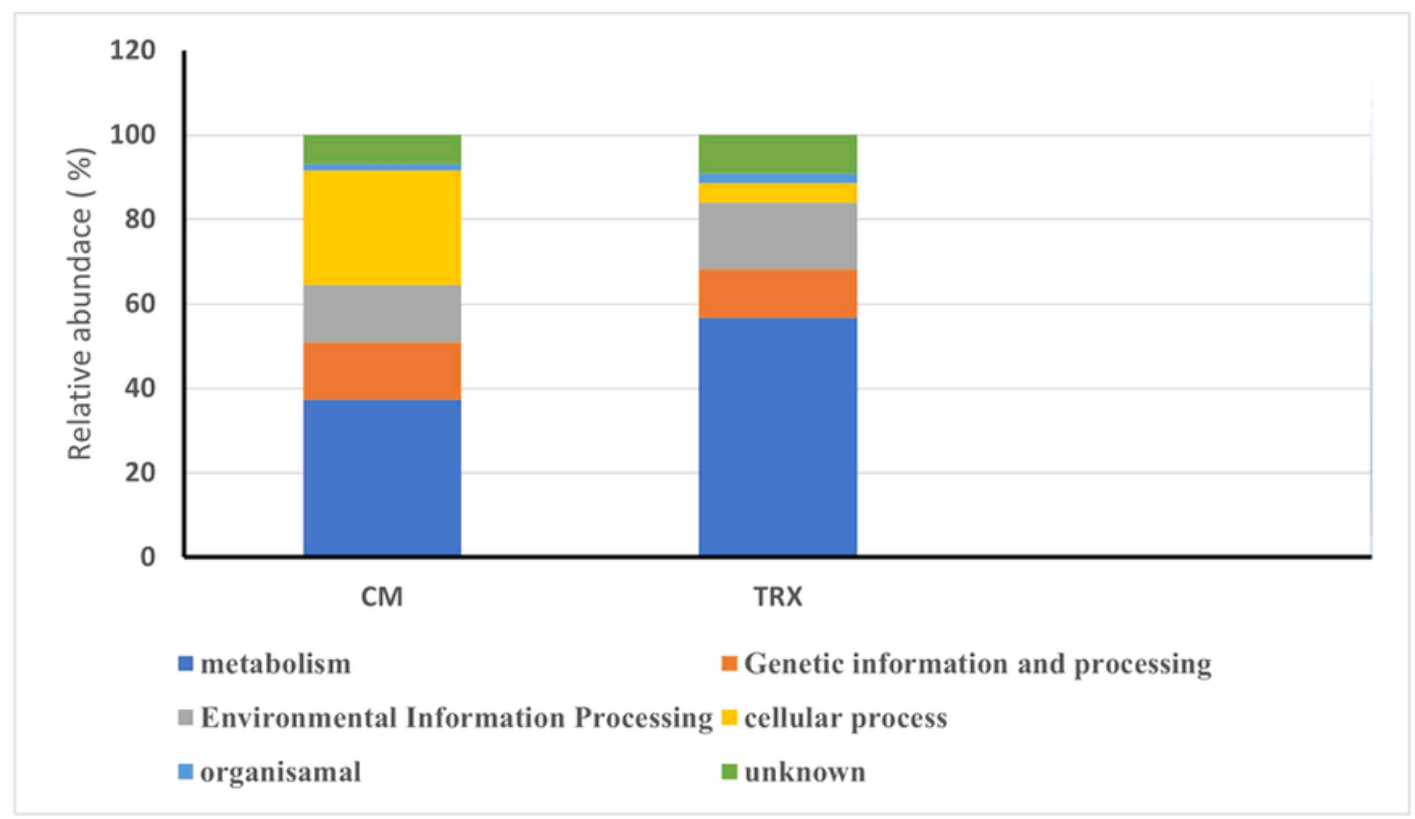

Figure 7

\section{Figure 7}

Shows the relative abundance of genes in $C$. odorata and T. procumbens soil. Carbohydrate metabolism was the most abundant metabolic pathway in $C$. odorata and $T$. procumbens soils. In $C$. odorata plants, the second abundant category was involved in cellular processes. Genes associated with genetic information and processing was expressed in C. odorata and T. procumbens species.

\section{Supplementary Files}

This is a list of supplementary files associated with this preprint. Click to download.

- supplementaryTable.1.docx

- supplementaryTable.2.docx 